\title{
Visual outcome after silicone oil removal and recurrent retinal detachment repair
}

\begin{abstract}
Purpose Prospective analysis of the effect of removal of silicone oil (ROSO) in eyes with complicated retinal detachments and evaluation of the visual outcome following recurrent retinal detachment after silicone oil removal.

Methods We evaluated 62 consecutive cases of ROSO over a 12 month period. All eyes had previously undergone silicone oil placement for complicated retinal detachments. All eyes undergoing scheduled ROSO over the time period of the study were entered and were reviewed post-operatively.

Results Sixty-two eyes were entered into the study. Twenty-one of 62 (34\%) developed recurrent retinal detachment following ROSO; 18 of these 21 recurrent detachments were reattached with one additional procedure and only 5 required replacement of the silicone oil. Ten of these 21 eyes (48\%) had improvement or stabilisation in final visual acuity compared with the presenting visual acuity. Overall, 39 eyes $(63 \%)$ had improvement in vision following ROSO and $76 \%$ of all eyes had ambulatory visual acuity at the end of the follow-up period. Fourteen eyes required cataract extraction with ROSO to attain this final visual acuity.

Conclusions Overall, ROSO has a significant rate of recurrent retinal detachment, with a high reattachment rate with one additional procedure. Few of these eyes need the silicone oil replaced. Most eyes will retain ambulatory visual acuity even with multiple procedures in cases of complicated retinal detachments following ROSO even with recurrent retinal detachment.
\end{abstract}

\section{C.J. Flaxel \\ G.W. Aylward \\ Moorfields Eye Hospital \\ City Road \\ London, EC1V 2PD, UK}

Christina J. Flaxel, MD Doheny Eye Institute University of Southern California

Keck School of Medicine

1450 San Pablo Street

Los Angeles

CA 90033, USA

e-mail: flaxelcj@aol.com.

\section{S.M. Mitchell}

Chelsea and Westminster Hospital

London, UK

Received: 22 February 2000 Accepted in revised form: 27 July 2000
Key words Ambulatory visual acuity, Complicated retinal detachment, Removal of silicone oil, Silicone oil

Silicone oil is used in the treatment of complex retinal detachments but late complications such as glaucoma and corneal decompensation are well known. ${ }^{1-4}$ The removal of silicone oil (ROSO) is advocated to reduce complications
CHRISTINA J. FLAXEL, over the long term. Once some complications have occurred, such as increased intraocular pressure, they may not be reversible even after silicone oil is removed. ${ }^{1}$ Risk of recurrent retinal detachment following ROSO is also significant, especially in the presence of proliferative vitreoretinopathy (PVR). ${ }^{5,6}$ The overall visual benefit of further surgical procedures to reattach the retina in those patients with recurrent detachments has not been studied. We separately evaluated the final visual and anatomical results in this group of patients who developed recurrent retinal detachments following ROSO and underwent further intraocular procedures for retinal reattachment in an attempt to determine whether further surgery on these eyes is beneficial in maintaining ambulatory visual acuity.

It is advocated by some that the use of $360^{\circ}$ indirect laser to the mid-peripheral retina approximately 6 weeks prior to ROSO or at the time of ROSO may decrease the incidence of recurrent retinal detachment. ${ }^{7,8}$ There are few prior data supporting this approach and we looked at those patients in our current series who underwent $360^{\circ}$ laser prior to or combined with ROSO.

\section{Methods}

Sixty-two consecutive eyes scheduled for routine ROSO following successful retinal attachment surgery over a 1 year time period were entered prospectively into this study. All eyes had been treated with injection of $1000 \mathrm{cS}$ silicone oil.

In addition to the placement of silicone oil, prior vitreoretinal procedures generally consisted of: 18 vitrectomies with intraocular gas tamponade (either $\mathrm{SF}_{6}$ or $\mathrm{C}_{3} \mathrm{~F}_{8}$ ), 5 of which included the placement of a scleral buckle; 15 scleral buckles with cryotherapy; 3 D-ACE (drain, air, cryotherapy, buckle) procedures; ${ }^{9}$ and 2 large horseshoe retinal tears treated with laser and/or cryotherapy only. In addition, revision of scleral buckle, membrane peeling and releasing retinotomy and retinectomy were carried out as indicated at the time of the last vitreoretinal procedure. All aphakic and 
pseudophakic eyes had surgical inferior peripheral iridectomies at the time of the initial placement of silicone oil.

Before silicone oil removal, all eyes had a complete ocular examination which included best-corrected Snellen visual acuity, measurement of intraocular pressure, slit-lamp examination with particular attention to the status of the cornea, lens and location of the silicone oil (anterior or posterior to the plane of the iris), and binocular indirect ophthalmoscopy.

ROSO was carried out for the following reasons: stable eyes with fully attached retina (48 eyes), chronic retinal detachment requiring further vitreoretinal surgery in conjunction with silicone oil removal (2 eyes), possible silicone-oil-related complications such as corneal oedema or ocular hypertension (12 eyes).

ROSO was performed under general or peribulbar anaesthesia utilising a posterior inferotemporal infusion cannula placed in a standard fashion. In phakic eyes, the oil was removed utilising a superior sclerostomy $4.5 \mathrm{~mm}$ posterior to the limbus and in aphakic and pseudophakic eyes the oil was removed through a $3 \mathrm{~mm}$ circumferential limbal incision. In 14 cases, ROSO was combined with cataract extraction and posterior chamber intraocular lens implantation.

Post-operative topical antibiotics, cycloplegics and corticosteroids were used in a standard fashion and tapered accordingly over a 4-6 week period. Complete ocular examinations including indirect ophthalmoscopy were carried out at 1 day, 1 week, 4 weeks, 3 months and 5 months post-operatively. We followed these eyes for an average of 12 months (range 8 months to 2 years) after ROSO for evidence of recurrent retinal detachment and other complications relating to the silicone oil, including persistent intraocular pressure elevation and corneal decompensation.

\section{Results}

THere were 48 men and 14 women. The ages ranged from 10 years to 80 years (average age 54 years). There were 34 right eyes and 28 left eyes; there were no bilateral cases. The primary diagnoses before oil placement included 45 (73\%) rhegmatogenous retinal detachment (RRD), 8 traction retinal detachments which included vascular (primarily diabetic) and posttraumatic retinal detachments $(12 \%)$, and 9 giant retinal tears $(15 \%)$. There was no statistically significant association between the primary diagnosis and the final retinal status following silicone oil removal (Fisher exact; $p<0.05)$. Twenty (32\%) of these detachments were initially macula on and $42(68 \%)$ were macula off. There was no statistically significant association between the initial macular status and the final visual acuity (Fisher exact; $p<0.05)$.

Twenty-five ( $44 \%)$ of these eyes had silicone oil placed as the primary procedure, while $20(33 \%)$ and had one prior surgical retina procedure. Seventeen (26\%) eyes had two or more prior vitreoretinal procedures. Sixtythree per cent of eyes (39 total) had silicone oil placed for PVR (grade B2 or worse) and in 11 of these (28\%), silicone oil was placed as the primary procedure. In 9 eyes $(15 \%)$, silicone oil was used for giant retinal tears and 8 of these had primary oil placement. The remaining 14 eyes $(22 \%)$ had silicone oil for a variety of other reasons which included 2 subretinal haemorrhages from drainage complications during scleral buckling surgery, 4 combined traction/rhegmatogenous retinal detachments, 3 post-traumatic retinal detachments, 3 eyes with multiple breaks in four quadrants, and 2 multiply recurrent vitreous haemorrhages. Six of these 14 eyes had silicone oil placed at the primary vitrectomy. We found no statistically significant association between

Table 1. Characteristics of eyes with recurrent retinal detachments

\begin{tabular}{|c|c|c|c|c|}
\hline \multirow[b]{2}{*}{ Eye no. } & \multirow[b]{2}{*}{ Presenting diagnosis (initial) } & \multicolumn{3}{|c|}{ Visual acuity } \\
\hline & & Initial & Before ROSO & Final \\
\hline 1 & Proliferative diabetic retinopathy, Traction retinal detachment & $\mathrm{CF}$ & $\mathrm{CF}$ & $\mathrm{CF}$ \\
\hline 2 & GRT, aphakic & $6 / 60$ & $6 / 60$ & $6 / 6$ \\
\hline 3 & High myopia, PVR & $6 / 24$ & $1 / 24$ & $6 / 36$ \\
\hline 4 & Recurrent inferior detachment & $6 / 36$ & $6 / 36$ & $6 / 60$ \\
\hline 5 & Aphakic, PVR & $6 / 60$ & $\mathrm{HM}$ & CF \\
\hline 6 & High myopia, PVR & HM & HM & HM \\
\hline 7 & PVR & $\mathrm{HM}$ & HM & PL \\
\hline 8 & PVR & $6 / 6$ & $\mathrm{HM}$ & NPL \\
\hline 9 & Myopia, PVR & $2 / 36$ & $2 / 36$ & CF \\
\hline 10 & Combined traction and rhegmatogenous retinal detachment & $6 / 60$ & $6 / 36$ & $6 / 24$ \\
\hline 11 & Aphakic & HM & CF & $6 / 60$ \\
\hline 12 & Aphakic, PVR & HM & $\mathrm{HM}$ & $3 / 60$ \\
\hline 13 & High myopia, PVR & HM & HM & PL \\
\hline 14 & GRT, pseudophakia & $6 / 12$ & $6 / 12$ & PL \\
\hline 15 & PVR & $6 / 60$ & $\mathrm{HM}$ & HM \\
\hline 16 & PVR & PL & PL & $1 / 36$ \\
\hline 17 & PVR & HM & $\mathrm{HM}$ & $\mathrm{HM}$ \\
\hline 18 & GRT & $6 / 18$ & $6 / 18$ & PL \\
\hline 19 & GRT & $6 / 6$ & $6 / 6$ & $\mathrm{CF}$ \\
\hline 20 & PVR & HM & CF & HM \\
\hline 21 & PVR & $6 / 36$ & HM & $6 / 24$ \\
\hline
\end{tabular}

PVR, Proliferative vitreoretinopathy; GRT, giant retinal tear.

$\mathrm{CF}$, count fingers; HM, hand motion; PL, perception of light only; NPL, no perception of light. 
Table 2. Retinal status

\begin{tabular}{lccc}
\hline Retinal status & Before ROSO & After ROSE & Final \\
\hline Attached & $60(97 \%)$ & $41(66 \%)$ & $59(95 \%)$ \\
Detached & $2(3 \%)$ & $21(34 \%)$ & $3(5 \%)$ \\
\hline
\end{tabular}

the number of vitreoretinal procedures and the final retinal status and either primary diagnosis and recurrent detachment following silicone oil removal or final retinal status (Fisher exact; $p<0.05$ ) (Table 1), though with the multiple causes of retinal detachment in this series, this result may not be relevant.

At the time of ROSO, 11 eyes (18\%) had evidence of elevated intraocular pressure prior to silicone oil removal and of these eyes, 4 were aphakic. All 11 eyes had persistently elevated intraocular pressure after silicone oil removal. Three of these 11 eyes developed recurrent retinal detachments; 2 of these 3 eyes were aphakic (eyes $3,12,19)$ (Table 1). Initial lens status included $12(19 \%)$ aphakic eyes, $40(65 \%)$ phakic eyes and $10(16 \%)$ pseudophakic eyes. There was no statistically significant association between initial lens status and either retinal status after ROSO or final retinal status (Fisher exact; $p<$ $0.05)$. Twenty-one eyes ( $35 \%$ ) developed cataracts related to the silicone oil and of these 14 required cataract extraction at the time of silicone oil removal. Six of these 14 eyes (43\%) developed recurrent retinal detachment after silicone oil removal. Two eyes had evidence of corneal decompensation or corneal oedema at the time of silicone oil removal. Two eyes had chronic, stable inferior retinal detachments without macular involvement at the time of silicone oil removal. One of these eyes (eye 4) had evidence of reproliferation and is now reattached following another procedure at the time of silicone oil removal with $\mathrm{SF}_{6}$ gas and without silicone oil replacement (Table 1). The second eye (eye 7), underwent two procedures with oil in situ including retinectomy and endolaser 3 months before ROSO. This eye had ROSO then performed for corneal decompensation and chronic inferior retinal detachment and is now phthisicial with PL vision and no further surgery was felt to be appropriate (Table 1).

Of the eyes that had silicone oil removed, 21 of 62 had recurrent retinal detachments (34\%) (Table 1). This 34\% includes the 2 eyes with chronic retinal detachments at the time of the removal of silicone oil (eyes 4 and 7), 3 eyes with elevated intraocular pressure (eyes 3, 12, 19), eye 7 also had corneal decompensation and the remaining 16 eyes in this group were felt to have stable attached retinae prior to ROSO. The length of time from silicone oil removal to recurrent retinal detachment varied from 1 day to 6 months but 18 of 21 redetachments occurred within 1 month of ROSO (86\%). Of the 21 recurrent retinal detachments, 20 eyes underwent 1 further procedure to stabilise the retina and 18 of these remain with the retina attached at the last follow-up (Table 2). Nine of these 18 attached eyes showed either stabilisation or improved visual acuity compared with the presenting visual acuity (Table 3). Only 5 of 20 required replacement of silicone oil but these 5 have oil remaining in situ at the last follow-up visit.

Twenty-seven eyes did not undergo cataract extraction with ROSO and did not develop recurrent retinal detachment. The visual acuity in this group of eyes prior to silicone oil placement ranged from $6 / 6$ to PL (average $\mathrm{CF}$ ), while following ROSO the average visual acuity improved to $6 / 60$ (range $6 / 6$ to HM). One patient had no visual acuity recorded in this group due to severe mental retardation.

Five eyes had $360^{\circ}$ laser performed approximately 6 weeks prior to $\mathrm{ROSO}(8 \%)$ and 1 eye had $360^{\circ}$ laser performed at the time of ROSO. Four of these eyes remained with the retina attached $(66 \%)$ and 2 detached $(33 \%)$.

Visual acuities are summarised in Table 3. Presenting visual acuities (at the time of the first retinal surgery) ranged from $6 / 6$ to PL (average visual acuity was CF). Visual acuity immediately prior to ROSO ranged from $6 / 6$ to PL (average visual acuity was CF). Post-ROSO visual acuities (representing the final visual acuity at last follow-up visit) ranged from $6 / 6$ to NPL (average visual acuity was CF). Overall, 39 eyes (63\%) showed improvement in visual acuity after ROSO at the last follow-up visit, 14 eyes showed worsening of visual acuity and 8 eyes showed no change in visual acuity. Forty-seven eyes $(76 \%)$ had ambulatory visual acuity of $\mathrm{CF}$ or better following the removal of silicone oil at the last follow-up visit. One patient had no visual acuity recorded during his care because he was severely mentally retarded, but by observation while he was hospitalised, he did obtain ambulatory visual acuity following ROSO. Of the eyes with recurrent retinal detachments, $57 \%$ (12 of 21 eyes) had ambulatory visual acuity at last follow-up (Fig. 1). Silicone oil had remained in the eyes from 27 days to 497 days (average 212 days or approximately 7 months). There was no statistically significant change in visual acuity from the presenting visual acuity prior to the first retinal detachment surgery to the final visual acuity at the last follow-up clinic visit (average 1 year after removal of silicone oil) (Fisher exact; $p<0.05)$; comparable visual acuity was maintained despite multiple surgical procedures.

Table 3. Visual acuity

\begin{tabular}{lcccccc}
\hline Visual acuity & $6 / 6-6 / 12$ & $6 / 18-6 / 36$ & $6 / 60-C F$ & HM & PL & NPL \\
\hline At presentation & 7 & 12 & 19 & 15 & 8 & 0 \\
Before oil removal & 4 & 6 & 21 & 22 & 8 & 0 \\
At last follow-up & 7 & 11 & 28 & 10 & 4 & 1 \\
\hline
\end{tabular}

$\mathrm{CF}$, count fingers; HM, hand motion; PL, perception of light; NPL, no perception of light.

${ }^{*}$ One patient had no visual acuity recorded due to severe mental retardation. 


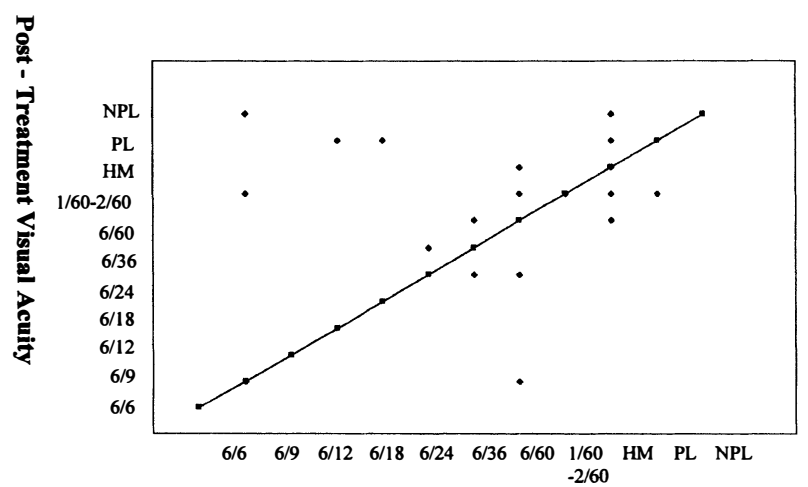

Pre - Treatment Visual Acuity

Fig. 1. Visual acuity before and after removal of silicone oil.

\section{Discussion}

Silicone oil has been used for complicated retinal detachments since the 1960s when it was first introduced by Cibis et al. ${ }^{10}$ Despite its many reported potential complications, ${ }^{1,2,4}$ silicone oil has become widely used in cases of recurrent retinal detachment due to PVR, giant retinal tears and other complicated retinal detachment procedures. ${ }^{4,8}$ Most surgeons who have long-term experience with silicone oil advocate its removal at some point in time $e^{1,4-8,11}$ to prevent the well-documented longterm complications of its use. ${ }^{1,4-8,11}$ One series by Caswell and Gregor ${ }^{2}$ recommended ROSO only in carefully selected cases, especially in those patients who are likely to develop severe anterior segment complications. They reported a $27 \%$ incidence of recurrent retinal detachment after oil removal.

The timing for ROSO is still controversial and varies, depending on the series, from 8 weeks and 12 weeks as advocated by Gonvers ${ }^{8}$ and Franks and Leaver, ${ }^{1}$ to 6 months as reported in the Silicone Oil Study Report 6, ${ }^{11}$ to 22 months as advocated by Hoing et al..$^{5}$ The timing of the removal does not appear to be correlated with the incidence of recurrent detachment, in our series or in other series. ${ }^{5,11}$ It is felt that silicone oil should remain in situ until a stable anatomical and functional status has been achieved and presumably the proliferative process has stopped. Such a stable situation is somewhat difficult to assess and is largely based on clinical appearances and surgeon experience. At our institution, we aim to remove silicone oil between 6 weeks and 6 months, depending on the underlying pathology and retinal status. It is removed earlier if any silicone-oil-related complications occur such as early corneal changes or raised intraocular pressure. The mean duration of silicone oil tamponade in our current series was 7 months; this is similar to that reported by the Silicone Study Group. ${ }^{11}$

The rate of recurrent retinal detachment in previous reports has varied from less than $1 \%$ to over $50 \% .{ }^{1-11}$ In this current series, we report an incidence of recurrent retinal detachment of $34 \%$ but we also note that of the 21 retinae that redetached, $18(86 \%)$ were re-attached with only one additional procedure and only 6 of these cases required re-infusion of silicone oil. This gives an overall success rate of $92 \%$, excluding the patient who refused further surgery and the 5 patients with oil in situ. Of these 18 patients, 10 showed stabilisation or improved visual acuity and only 5 cases still have oil in situ; 12 of these eyes $(67 \%)$ have maintained functional visual acuity of CF or better. Fourteen of 21 redetachments occurred within 1 week of ROSO and 18 of 21 occurred within 3 weeks of ROSO. Other series have reported that recurrent retinal detachments after ROSO occur within 6 months of the procedure and often within 3 months. ${ }^{6,11}$ However a recent report by Jonas et al. ${ }^{12}$ evaluated the timing of retinal redetachment after ROSO and found that the greatest risk or redetachment was within 50 days of oil removal with the risk decreasing sharply after 3-5 months. The short period of time between the ROSO and recurrent detachment would implicate either residual anterior retinal traction or opening of old breaks that had been tamponaded by the silicone oil, or possibly new breaks from intraocular manipulations. In this particular series, we did not perform extensive intraocular manipulations during ROSO. Zilis et al ${ }^{6}$ postulated that the presence of the large silicone bubble filling most of the posterior cavity could lead to the concentration of proliferative factors at the retinal surface. This would lead to proliferation along the retinal surface which would be very difficult to see with the oil in place. Once the oil was removed, the proliferation would extend, leading to further traction and eventually recurrent detachment. There is also some evidence that silicone oil may stimulate release of mitogenic proliferative factors. ${ }^{12}$ Elimination of residual preretinal traction at the time of oil removal would also be expected to reduce the risk of redetachment. When necessary, we attempt to remove all residual epiretinal membranes at the time of ROSO. In this current series, no extensive posterior segment intervention was necessary. It may be that reinforcement of the choroidal adhesions either prior to oil removal or at the time of oil removal by indirect laser could decrease the rate of recurrent detachments. In 6 cases, $360^{\circ}$ indirect laser to the mid-peripheral retina was performed prior to or the time of ROSO. Two of these cases redetached.

These numbers are too small to appreciate any trends as to the advantages of laser versus potential

disadvantages.

There are several potential reasons for our high redetachment rate, not the least important of which is that we are a tertiary referral centre so even the primary detachments referred to us are complicated and this accounts for the high rate of silicone rate placement with the initial surgery. We also are referred detachments that have been initially operated elsewhere and failed. These cases have a high incidence of grade B or higher PVR. In other large-scale studies of silicone oil removal, such as the Silicone Oil Study, they admit that they were highly selective in choosing the eyes which had silicone oil removed. They did not remove oil from eyes that had the most severe PVR prior to oil placement even if the retina remained anatomically attached with oil in situ. In contrast, we do not necessarily use the severity of the pre-oil PVR alone in determining the timing of oil 
removal. Factors that we consider are the length of time the oil has been in place, the number of prior procedures, the presence of a scleral buckle, whether or not a break was found at the last procedure, adequate treatment of all breaks, presence or absence of retinal traction of detachment, presence of epiretinal proliferation, and presence of hypotony. Unfortunately, with multiple surgeons in a large teaching institution, the authors were not able personally to view each case intra-operatively to determine the cause of the recurrent retinal detachment after ROSO.

As noted in other series, visual acuity improved in the majority of eyes following silicone oil removal. ${ }^{1,11}$ More patients had ambulatory or better visual acuity after oil removal than had that level of visual acuity before their initial surgical procedure. This has also been reported in other series. ${ }^{6,11}$ It has been postulated by Zilis et al. ${ }^{6}$ that the improvement in visual acuity following ROSO is due to a combination of factors including the slow recovery of the naturally reattached retina, altered optics during silicone oil tamponade with improved refraction after silicone oil removal and possible silicone oil retinal toxicity with improved function after silicone oil removal. If the retina remains attached after successful silicone oil removal, progressive visual acuity loss is rare. We have found that comparable final visual acuity is maintained despite multiple vitreoretinal procedures, thus indicating that all attempts should be made to reattach these retinae even after multiple recurrent retinal detachments.

Other studies have also reported a significant incidence of cataract formation. ${ }^{2,3}$ Fourteen of our patients underwent cataract extraction at the time of ROSO but 6 (43\%) of these patients developed recurrent retinal detachment. A recent series showed no increased incidence of retinal detachment following cataract extraction after retinal detachment surgery, ${ }^{14}$ but these cases were not necessarily complicated retinal detachments and none involved silicone oil.

Eleven patients $(18 \%)$ had chronic elevated intraocular pressure following ROSO, which is somewhat less than the rate noted in prior articles. ${ }^{1,7}$ Other articles did have a larger number of aphakic eyes with silicone oil, which is known to be a risk factor for chronic pressure elevation following oil removal. We had no significant complications related to corneal decompensation or hypotony following silicone oil removal.

\section{Conclusions}

We realise the limitations of this study, which includes a wide variety of cases with multiple aetiologies, and acknowledge that the reasons we may not have found statistically significant differences on multiple variables is that the power of the study is too small to demonstrate these differences. However, we feel that we can make several guarded statements based on our data including observation that, following ROSO, stabilisation of visual acuity and improvement of visual function is seen even if recurrent retinal detachment occurs. Most cases of recurrent detachment can be repaired with only one additional procedure, often without the use of silicone oil. The recurrent retinal detachment rate is high and an attempt should be made to discern the aetiology of these recurrent retinal detachments at the time of the detachment repair to determine possible preventive measures in the future. We feel there are definitely limitations to the number of surgeries that should be performed in these cases and clinical judgement should be used in these complicated cases when determining whether it is acceptable from a visual and/or functional standpoint to proceed with further surgeries.

The use of pre-operative $360^{\circ}$ indirect laser to the midperipheral retina 6 weeks prior to ROSO may be of benefit in preventing recurrent retinal detachment, but the small numbers in this series preclude our making a definitive suggestion as to its use. Further controlled trials into the use of indirect laser either before silicone oil removal or at the time of removal are currently under way.

\section{References}

1. Franks WA, Leaver PK. Removal of silicone oil: rewards and penalties. Eye 1991;5:333-7.

2. Caswell AG, Gregor ZJ. Silicone oil removal. I. The effect on the complications of silicone oil. Br J Ophthalmol 1987;12:893-7

3. Caswell AG, Gregor ZJ. Silicone oil removal. II. Operative and postoperative complications. Br J Ophthalmol 1987;12:898-902.

4. Le Mer Y, Renard Y, Ameline B, Haut J. Long-term results of successful surgical treatment of retinal detachment by vitrectomy and silicone oil injection: effect of removal of the tamponade on further complications. J Fr Ophtalmol 1992;5:331-6.

5. Hoing C, Kampik A, Heidenkummer HP. Possibilities of silicone oil removal after complex vitreoretinal surgery. Fortschr Ophthalmol 1991;6:593-7.

6. Zilis JD, McCuen BW, de Juan E, et al. Results of silicone oil removal in advanced proliferative vitreoretinopathy. Am J Ophthalmol 1989;108:15-21.

7. Haut J, Besson D, Larricart P, LeMer Y. Results of early removal of intra-ocular silicone after vitreoretinal surgery. $J$ Fr Ophtalmol 1988;11:723-6.

8. Gonvers M. Temporary use of intraocular silicone oil in the treatment of detachment with massive periretinal proliferation: preliminary report. Ophthalmologica $1982 ; 4: 210-8$.

9. Gilbert C, McLeod D. D-ACE surgical sequence for selected bullous retinal detachments. Br J Ophthalmol 1985;10:733-6.

10. Cibis PA, Becker B, Okun E, Cowan S. The use of liquid silicone in retinal detachment surgery. Arch Ophthalmol 1962;681:590-9.

11. Hutton WL, Azen SP, et al. The effects of silicone oil removal. Silicone Study Report 6. Arch Ophthalmol 1994;6:778-85.

12. Jonas JB, Budde WM, Knorr HLJ. Timing of retinal redetachment after removal of intraocular silicone oil tamponade. Am J Ophthalmol 1999;128:628-31.

13. Lambrou FH, Burke JM, Aaberg TM. Effects of silicone oil on experimental traction retinal detachment. Arch Ophthalmol 1987;105:1269-72.

14. Kerrison JB, Marsh M, Stark WJ, Haller JA. Phacoemulsification after retinal detachment surgery. Ophthalmology 1996;103:216-9. 